\title{
Laser ignitibility of energetic crystals doped with gold nanoparticles
}

\author{
Sam Churchyard, Xiao Fang*, Ranko Vrcelj
}

Centre for Defence Chemistry, Cranfield University, Defence Academy of the UK, Shrivenham SN6 8LA, UK.

* Corresponding author. Email: x.fang@cranfield.ac.uk

\begin{abstract}
Laser ignition mechanism enhances the safety of explosive applications. However, optical sensitisation of the energetic materials is required for their optical absorption. In this research, explosive Cyclotrimethylene Trinitramine (RDX) was doped with gold nanoparticles during recrystallisation to provide a suitable optical sensitisation method. The relationship between the precipitation rate used during RDX recrystallisation and the subsequent laser ignition properties using an 808-nm continuous wave diode laser was studied. Faster initial precipitation was found to reduce the laser ignition delay times and smaller nanoparticle size reduced ignition thresholds. It was shown that recrystallized RDX particle size, which is determined by both nanoparticle induced nucleation and precipitation rate, affects the ignition delay time and quality of the crystals. The largest crystals in each batch were investigated using both high-speed photography and microscopic etching, revealing that in contrast to the bulk sample (which was generally a powder), a slower precipitation seems to achieve better doping. The largest crystals in each sample therefore do not represent the bulk sample, a useful indication as such large crystals are easier to handle and therefore tend to be selected for detailed characterisation work.
\end{abstract}

Keywords: RDX precipitation; nanoparticles; plasmon resonance; laser ignition

\section{Introduction}

In the field of energetics, plasmon resonances of nanoparticles lead to useful radiative properties which can affect bulk performance of an energetic material, however, trying to appropriately site suitable nanoparticles within an energetic material can cause downstream processing effects. Current explosive ignition systems use a traditional three-step explosive train as shown in Figure 1. The detonator typically contains sensitive primary explosives, which are easy to ignite, but have many issues associated with their use. As their name implies, primary explosives are the most sensitive of explosives, in particular with respect to physical insults, such as impact. Lead azide, a typical and widely used military primary explosive [1], raises multiple safety concerns because of its sensitivity and chemical instability [2]. Lead azide is much more sensitive than the secondary explosive RDX, with Rotter Impact Test figures of insensitivity (Fol) values of 20 and 80 respectively [3]. Most detonators utilising primary explosives are electric bridgewire types, which are susceptible to accidental ignition if handled roughly or exposed to strong electromagnetic (EM) radiation, questioning their use in many applications $[4,5]$. Furthermore, many primary explosives contain objectionable heavy metals and are harmful to the environment and thus restricted by legislation, creating a demand for safer and cleaner alternatives [6]. 


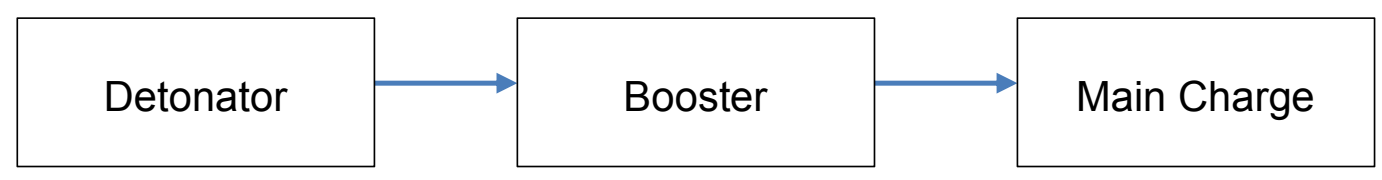

Figure 1. The traditional explosive train.

While the search for replacement primary explosives continues, other avenues for detonation are being investigated and one particularly promising solution is laser ignition. A detonation system based on this principle would not require primary explosives, rendering them much safer than traditional systems and offers the possibilities of removing the more objectionable materials, such as lead based compounds. Furthermore, laser detonators do not require a direct electrical connection to the energetic material, and are therefore much less susceptible to EM radiation insults.

In the case of RDX, ignition depends on its optical absorbance at a given wavelength. RDX absorbs strongly in the ultraviolet (UV) region of the EM spectrum [7]. Work by Renlund et al demonstrated that RDX can be ignited by a 24-ns pulse from a 308-nm laser, but not by a 20-ns pulse from a 1064-nm laser [8], and work by Yan et al described the ignition of a single RDX crystal by a 6.4-ns pulse from a 355$\mathrm{nm}$ UV laser, with radiant energy densities of $2.30-5.5 \mathrm{~J} \mathrm{~cm}^{-2}$ required for $100 \%$ ignition dependent upon which face is irradiated $[9,10]$. For this application, a major issue for UV lasers is that they are bulky and expensive, making them unsuitable for detonator applications, whereas diode lasers, which operate in the infrared-to-visible (IR-Vis) region, are small and inexpensive, thus much preferred [11].

Visible (diode) lasers can ignite pure RDX but require long ignition times due to the low absorbance in this region. McGrane and Moore showed that a continuous wave (CW) 532-nm laser operating for $120 \mathrm{~s}$ would ignite RDX at powers greater than 0.6 W, but ignition did not occur at all with a 1550-nm IR laser operating in the same manner, although melting and decomposition did occur at 1.4 W [12]. The direct ignition of pure RDX by IR-Vis lasers is therefore not practical for detonator applications.

As RDX does not absorb well in the IR-Vis region, optical sensitization is required to produce a practicable detonator. Carbon black is suitable for this purpose, with Fang and McLuckie showing that ignition of 1,1-diamino-2,2-dinitroethene (FOX-7) is promoted by carbon black using a 974-nm CW laser with a minimum power of $10 \mathrm{~W}$ [13]. Ignition delay was reduced to $70 \mu \mathrm{s}$ at a power of $40 \mathrm{~W}$. Fang and Ahmad showed a similar effect for the explosive Hexanitrostilbene (HNS), where pure HNS is not ignited by an 808-nm CW laser even at $45 \mathrm{~W}$, but with carbon black the threshold was reduced to $4 \mathrm{~W}$ [14]. Recently also Gillard showed the ignition of a propellant mainly composed of RDX by a diode laser with power $1.43 \sim 10 \mathrm{~W}$ [15]. One disadvantage of carbon black is that it absorbs energy efficiently over a broad spectrum, making it susceptible to interference from accidental or even hostile light sources.

Laser-absorbing dyes perform similarly to carbon black, reducing the HNS ignition threshold to $4 \mathrm{~W}$ for the same 808-nm laser, but has a narrow absorbance band at 
$811 \mathrm{~nm}$, making it less susceptible to interference [14]. Interestingly, the dye absorbs energy more efficiently than carbon black at this wavelength but the two sensitisers achieved a similar performance, suggesting that the thermal properties of the sensitiser also play an important role in the ignition process.

More recently, Fang et al have shown that gold nanoparticles (GNPs) also act as sensitizers. RDX doped with $0.5 \%$ (w/w) GNPs can reduce the ignition threshold from no ignition at $45 \mathrm{~W}$ to $100 \%$ ignition at $2 \mathrm{~W}$ using an 808-nm CW diode laser [16]. The RDX crystals were doped with GNPs by fast-precipitation crystallisation using an antisolvent. However, the quality of the nanoparticle doping achieved using this method is unclear, and the relationship between the strength of the precipitation and ignitibility is unknown.

Nanoparticles work as sensitizers due to surface plasmon resonance (SPR), where the sea of free electrons in nano-metals is induced to collective oscillation by incident light. The frequency of oscillation coincides with a particular frequency of incident light and resonance occurs leading to high amplitudes of oscillation, inducing strong, localised EM fields around the nanoparticle surface giving them their desirable radiative properties. Gold is superior at wavelengths greater than $600 \mathrm{~nm}$ and is extremely stable whereas other materials such as silver and aluminium are prone to oxidation, which will negatively impact plasmon resonance [17-19].

For the chosen nanoparticles to cause an effect, they need to be incorporated into the crystalline matrix, allowing the absorbed optical energy to be converted to heat, and effectively acting as a hotspot. This incorporation into the crystal (as opposed to simply lying on the surface) is necessary as it aids heat transfer into the bulk of the explosive and reduces the heat loss during ignition. There are several methods of doping nanoparticles into crystals but the simplest technique for doping nanoparticles inside a single crystal, and the one to be used in this present work, is to use a rapid precipitation as used by Reece et al to successfully dope nanoparticles into Ammonium Perchlorate crystals to improve propellant performance [20].

Laser ignition by cheap, low power IR CW lasers is a desirable technology to improve safety and this work investigates the effect of GNPs on both the ignition characteristics of GNPs doped RDX and also upon the crystallisation behaviour.

\section{Experimental}

Materials: The RDX used in this work was Type 1, <1 \% HMX. The nanoparticles used were all citrate coated gold nanorods (NanoPartz, Loveland, CO, USA) and were of two sizes, the smaller were $10 \times 40 \mathrm{~nm}$ and the larger were $40 \times 160 \mathrm{~nm}$. The acetone was Laboratory Reagent grade from Fisher Scientific UK. The anti-solvent used for all experiments was de-ionised water. The pipettes used were Rainin pipettes by Mettler Toledo with an accuracy of $\pm 0.5 \mu \mathrm{l}$.

Crystallization and doping RDX crystals with GNPs: A stock solution of RDX was produced by dissolving $190 \pm 5 \mathrm{mg} \mathrm{RDX}$ in $4 \mathrm{ml} \pm 5 \mu$ lacetone at the room temperature of $296 \mathrm{~K}$, which was slightly under-saturated as the solution saturates at temperature of $\sim 288 \mathrm{~K}$. To this stock RDX/acetone solution, GNPs were added to achieve $0.5 \%$ (wt.) doping, prior to usage, thus the total amount of RDX plus nanoparticles was the same for all samples. 
Single crystals: For large single crystals doped with GNPs, the stock solution was transferred into a crystallisation dish, covered with parafilm with 6 pinholes pierced to permit slow evaporation of the acetone.

Precipitation: Recrystallisation through precipitation by addition of anti-solvent (deionised water) containing GNPs, was induced at room temperature to achieve two different Solvent:Antisolvent (S:AS) ratios, 16:1 and 4:1. The required volume of the anti-solvent was added to the RDX/acetone solution to achieve the correct S:AS ratio. For the single-stage crashes, the entire RDX solution in acetone was mixed with the entire water volume containing GNPs as anti-solvent. To examine the role of precipitation rate, two-stage precipitation was used by initially achieving 1:1 and 1:3 $\mathrm{S}$ :AS ratios before the addition of more RDX/acetone solution in order to sum the amounts to give a final 4:1 ratio. To do this, the required amount of RDX/acetone was pipetted into the anti-solvent, mixed and stirred, before the rest of the RDX/acetone solution was added. These samples are given in Table 1.

Table 1. The GNP-doped samples with GNP sizes (diameter $x$ length): $10 \times 40 \mathrm{~nm}$ (small rods) and $40 \times 160 \mathrm{~nm}$ (large rods).

\begin{tabular}{lcc}
\hline $\begin{array}{l}\text { Ratio (S:AS) } \\
\text { Initial/final ratio (for two stage } \\
\text { process) }\end{array}$ & $\begin{array}{c}\text { Anti-solvent required } \\
(\mathbf{m l})\end{array}$ & Nanoparticles used \\
\hline $16: 1$ & 0.25 & Large \\
$4: 1$ & 1 & Large \\
$1: 1 / 4: 1$ (two-stage) & 1 & Large \\
$1: 3 / 4: 1$ (two-stage) & 1 & Large \\
$1: 3 / 4: 1$ (two-stage) & 1 & Small \\
\hline
\end{tabular}

Samples: Five samples were produced using the S:AS ratios and shown in Figure 2. The images show two effects evident from the precipitation, the general decrease in crystallite size with lower S:AS ratios (i.e. faster initial crash precipitation) and also the darkening of colour of the crystals with lower S:AS ratios. The decrease in crystallite size is to be expected from general crystallisation behaviour. As the amount of added antisolvent increases, the solubility of RDX in the mixed (miscible) solvents will decrease and move the solution into the labile region, leading to both increased nucleation and faster growth, thus to more and smaller crystals. Similarly, the increased nucleation and growth rates mean that the GNPs are more likely to be incorporated into the bulk of the RDX, either as a nucleation site, or simply as an impurity. It is notable that in Figure 2, there is little difference in the colour of the samples in Figure $2 b-d$ (and arguably 2e), but the sample colour in Figure $2 a$ is appreciably darker than the rest. This is because that there were more GNPs in the sample of Figure 2a due to their smaller sizes when the five samples had the same concentration by weight of GNPs, and also individual smaller GNPs optically absorb more. It is notable that the two-step precipitation still generates small crystals, with the initial addition of anti-solvent driving the nucleation and growth, followed by a slower 
growth, when the second addition of solvent occurs. For the faster initial precipitations, the majority of the crystals were less than $200 \mu \mathrm{m}$ in diameter, with very few achieving $\sim 1 \mathrm{~mm}$, whereas, many more crystals from the slower single step precipitations (whether 4:1 or 16:1) were larger than $1 \mathrm{~mm}$ and often $2-3 \mathrm{~mm}$ in size.

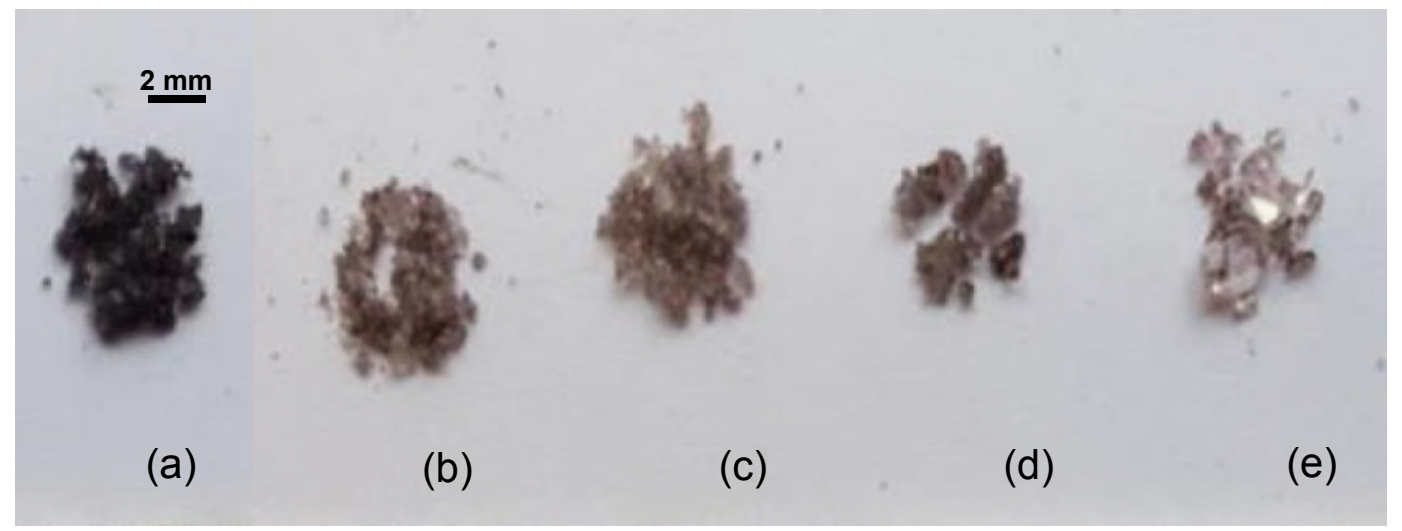

Figure 2. The GNP doped RDX crystal samples. (a) Small GNP 1:3/4:1. (b) Large GNP 1:3/4:1. (c) Large GNP 1:1/4:1. (d) Large GNP 4:1. (e) Large GNP 16:1. The scale bar is 2 $\mathrm{mm}$.

Etching: Etching was performed by simply stroking an RDX crystal on an acetone wetted filter paper on a glass polishing plate held at $296 \mathrm{~K}$.

Optical microscopy: Optical microscopy was conducted using a Leica DMLM microscope with a Qimaging QiCam Fast 1394 camera.

Ignition threshold and delay: $3 \mathrm{mg}$ powder samples were loaded into a sample holder consisting of an aluminium block with 10 holes ( $3 \mathrm{~mm}$ diameter, $3 \mathrm{~mm}$ deep). The sample in one of the holes was manually tamped and irradiated by a focused laser beam for both ignition delay and ignition threshold tests. The initiating diode laser (Jenoptik, JOLD-X-CPXF, Jena, Germany) was operated at $808 \mathrm{~nm}$ in CW mode for $0.3 \mathrm{~s}$ with a power of up to $45 \mathrm{~W}$ and focussed through a lens with focal length of 50 $\mathrm{mm}$. The beam width at the sample was $\sim 1 \mathrm{~mm}$. The general layout of the system and its scheme are shown in Figure 3. 

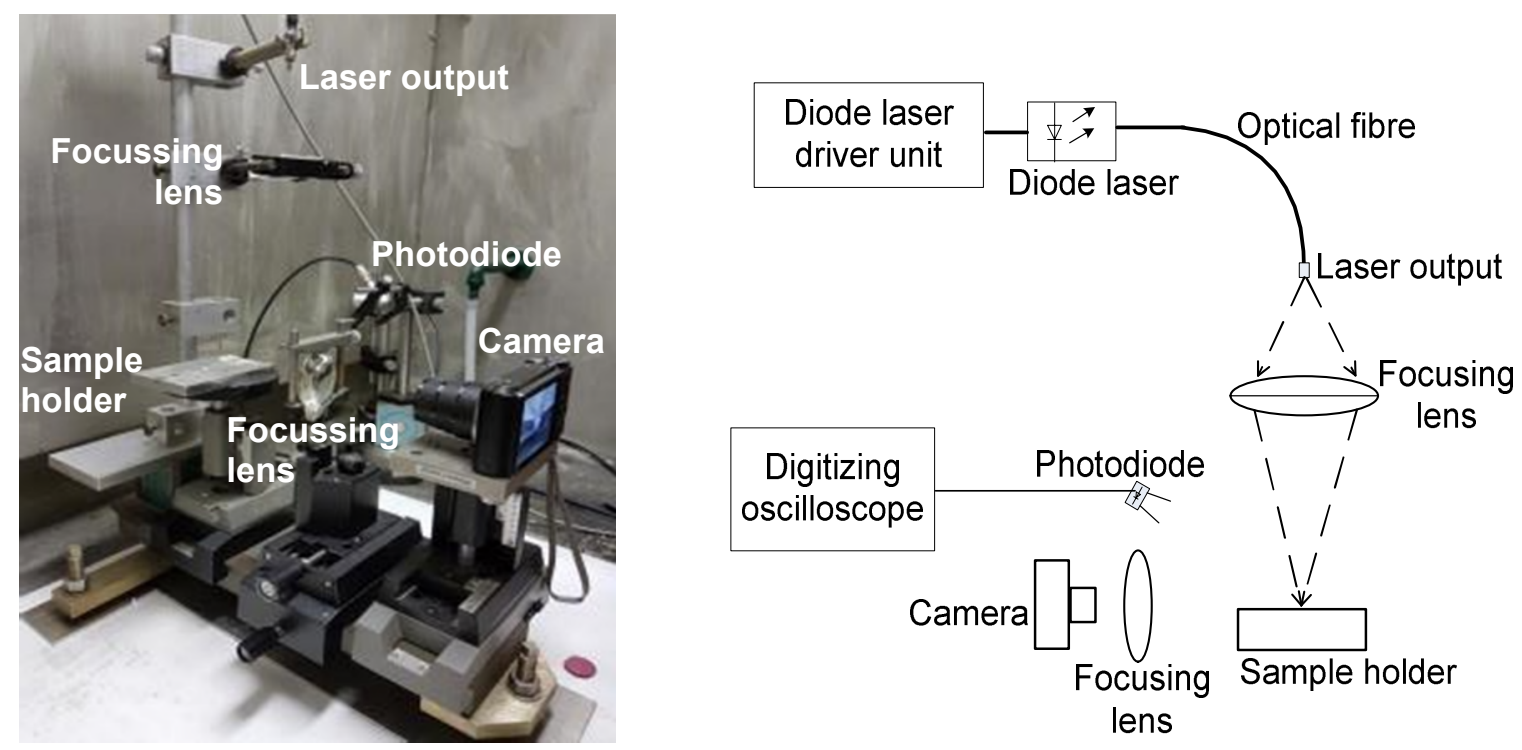

Figure 3. Apparatus for ignition experiments and its scheme.

For the threshold tests to compare the minimum laser powers required for ignition for the different samples, the ignition test was deemed positive if visual inspection revealed damage after irradiation. Laser powers were used at 5, 6 and $7 \mathrm{~W}$ for Small GNP 1:3/4:1 sample, 8 and 9 W for both Large GNP 1:3/4:1 and 1:1/4:1samples, and 9, 10, $12 \mathrm{~W}$ for Large GNP 4:1 sample. The test started at a lower laser power with increasing to the next higher power until 5 or more ignitions achieved out of the 7 repeated tests (i.e. an estimate of over $70 \%$ ignition rate). Ignition delay was measured under full laser power ( $45 \mathrm{~W}$ ) for all types of samples to compare their quickest ignition with the available maximum laser power in the study. This was done by using a photodiode (OSRAM Silicon PIN Photodiode BPX 65) connected to a DSO1024A oscilloscope (Agilent Technologies, Santa Clara, CA, USA) to detect the optical signal of the flame ignited by the laser. A typical delay trace is given in Figure 4.

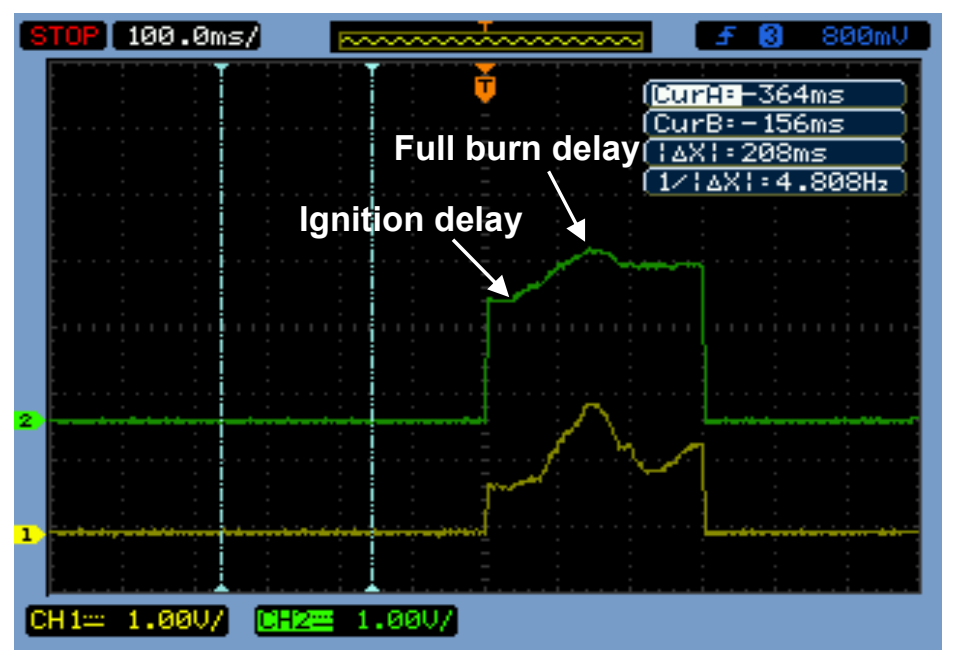

Figure 4. Oscilloscope signal traces for the measurement of ignition and full burn delay.

Effect of crystal size: Large crystals ( $\geq 1 \mathrm{~mm}$ ) with an average mass of $\sim 3.5 \mathrm{mg}$ were selected to make the images as clear as possible. The crystals were confined in 
a three-sided sample holder $3 \mathrm{~mm}$ in width to maintain visibility. The laser was operated in the same way as described for the ignition delay and threshold tests, but at 45 W. Data was collected using a Casio EX-ZR800 operating at 1000 frames per second and a $50-\mathrm{mm}$ lens was used to provide extra magnification. The software used for video analysis was VLC Media Player v2.2.6. The ignition delay was measured using frame-by-frame analysis instead of using the photodiode due to the ignition signal being too weak to be measured.

\section{Results and discussion}

\subsection{Microscopy}

Large crystals: The large crystals showed a typical morphology for RDX, with major (210) faces dominating the structure. Figure 5 shows the (210) natural external surfaces of the crystals. Figure $5 a$ shows a pure RDX crystal surface, which exhibits a banding, most likely due to macrostep formation on the surface of the crystal as part of the growth process. Figures $5 b-d$ show the (210) surface with low S:AS ratios. Figure $5 b$ contains small GNPs and it is similar to that seen in Figure $5 a$, with some colouring within the image, caused by GNPs on the surface. It is noteworthy that apart from the colouring effect, the surface looks similar to that of a pure RDX crystal in Figure 5a, with some large macrosteps apparent. Figures $5 \mathrm{c}$ and $\mathrm{d}$ show the low S:AS ratios for large GNPs. In this case, in addition to the appearance of GNPs at the surface, the macrosteps appear to be greatly enhanced in step height, which can be ascribed to their formation caused by the adherence of the large GNPs to the surface. This creates greater strain in the crystal, which ultimately can lead to cracking, as can be seen at the edge of the crystal in Figure $5 \mathrm{c}$. These larger macrostep heights and cracks within the crystal can also be seen in crystals with higher S:AS ratios (Figures $5 e, f)$. 

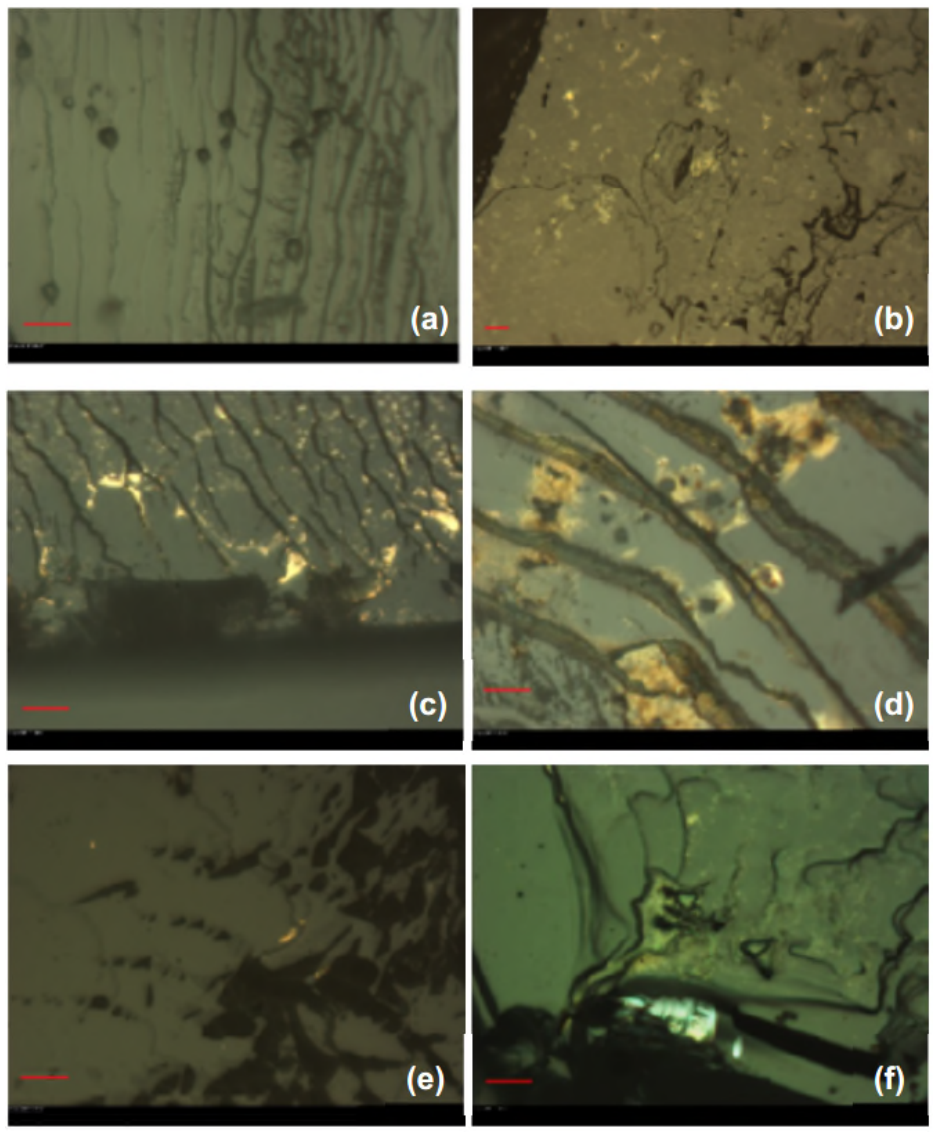

Figure 5. Microscopy of un-etched crystals representing (a) pure RDX; (b) 1:3/4:1 small GNP sample; (c) 1:3/4:1 large GNP sample; (d) 1:1/4:1 large GNP sample; (e) 4:1 large GNP sample; (f) 16:1 large GNP sample. Scale bars $=10 \mu \mathrm{m}$.

Etched large crystals: The same large crystals were then etched to reveal the inside of the crystal matrix, as shown in Figure 6a-c. These show that once the crystal surface was removed, large GNPs can be seen, in particular for Figure 6c, where the etch pits are clearly visible and that they contain GNPs is also evident. This shows that in addition to GNPs acting as nucleation sites, they are also incorporated into the RDX matrix as impurities, even for the slowest growing, high S:AS ratios, which are those to most likely exclude the GNPs as impurities. 

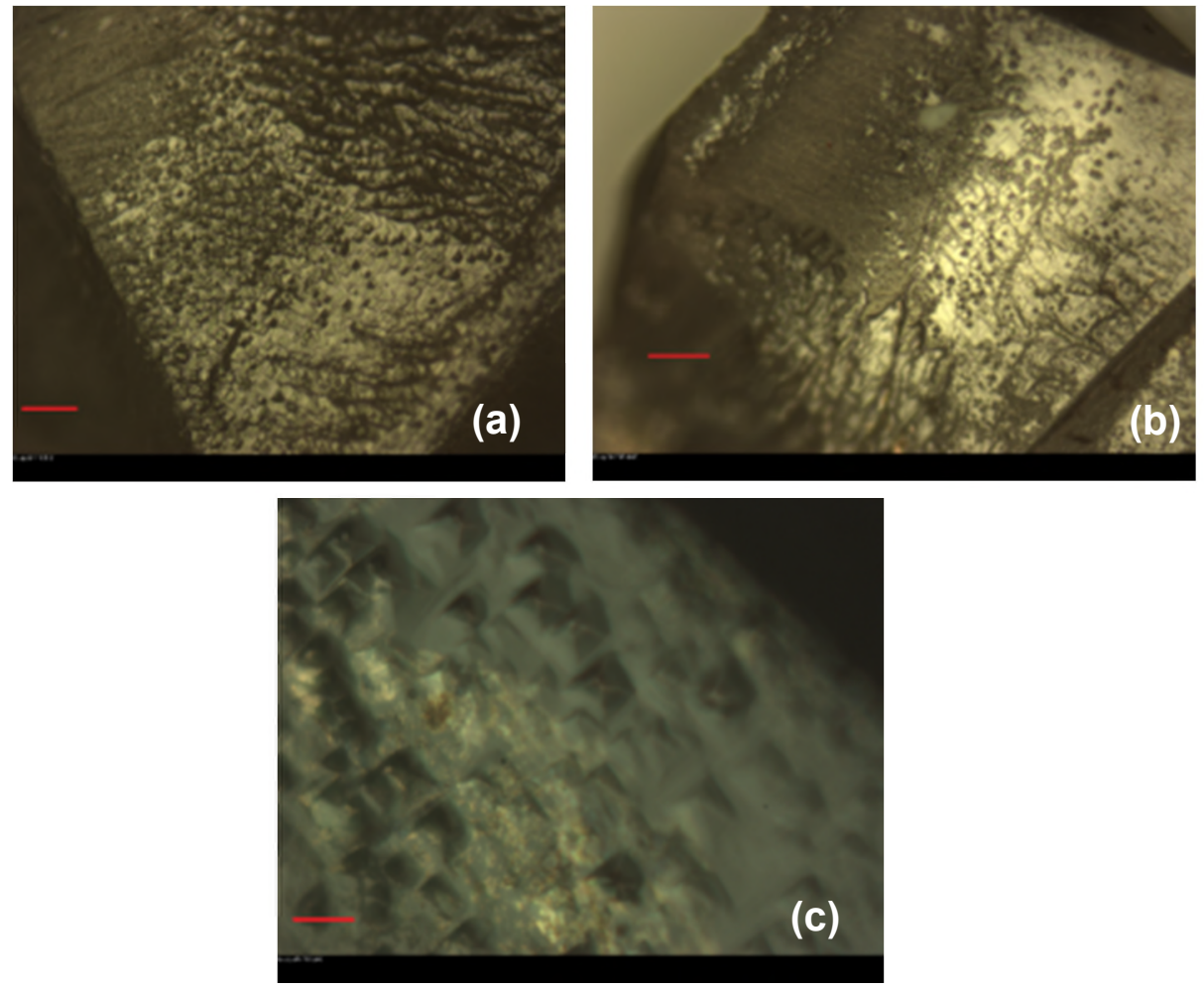

Figure 6. Microscopy images of etched crystals; (a) 1:1/4:1 large GNP sample; (b) 4:1 large GNP sample; (c) 16:1 large GNP sample. Scale bars $=10 \mu \mathrm{m}$.

The cracks in the RDX single crystals only occur within the crystals doped with large GNPs $(40 \times 160 \mathrm{~nm})$. The crystal structure of RDX is orthorhombic, with Space group Pbca and unit cell parameters of $a=1.3182 \mathrm{~nm}, b=1.1574 \mathrm{~nm}$ and $c=1.0709$ $\mathrm{nm}, V=1.63 \mathrm{~nm}^{3}$ so for the small GNPs $(10 \times 40 \mathrm{~nm})$, these have a volume of $\sim 3100$ $\mathrm{nm}^{3}$ and will individually represent an impurity of volume size 2000 greater than a unit cell. For a large GNP, the individual volume is $\sim 2 \times 10^{5} \mathrm{~nm}^{3}$ and thus an impurity has a volume of $\sim 1.23 \times 10^{5}$ times greater. Incorporating both will cause strain within the crystal, but certainly the latter will most likely distort the structure so much that it is not surprising that cracking occurs. The cracks are most obviously perpendicular to [001], but also parallel to [001] (e.g. in Figure 6c), which agrees well with the work of Hagan and Chaudhri [21] and Halfpenny et al [22]. However, only the (210) faces could be observed. Given that the size of the small GNP is approximately equal to that of the caxis, it would be of interest to see in the future if there is any direct crystallographic relationship between the small GNP and the lattice, as shown for spherical GNPs by Murugesan et al [23].

\subsection{Laser ignition}

Ignition delay: Figure 7 shows the results of the ignition delay tests for the doped RDX, showing the non-linear relationship between precipitation and delay time. 


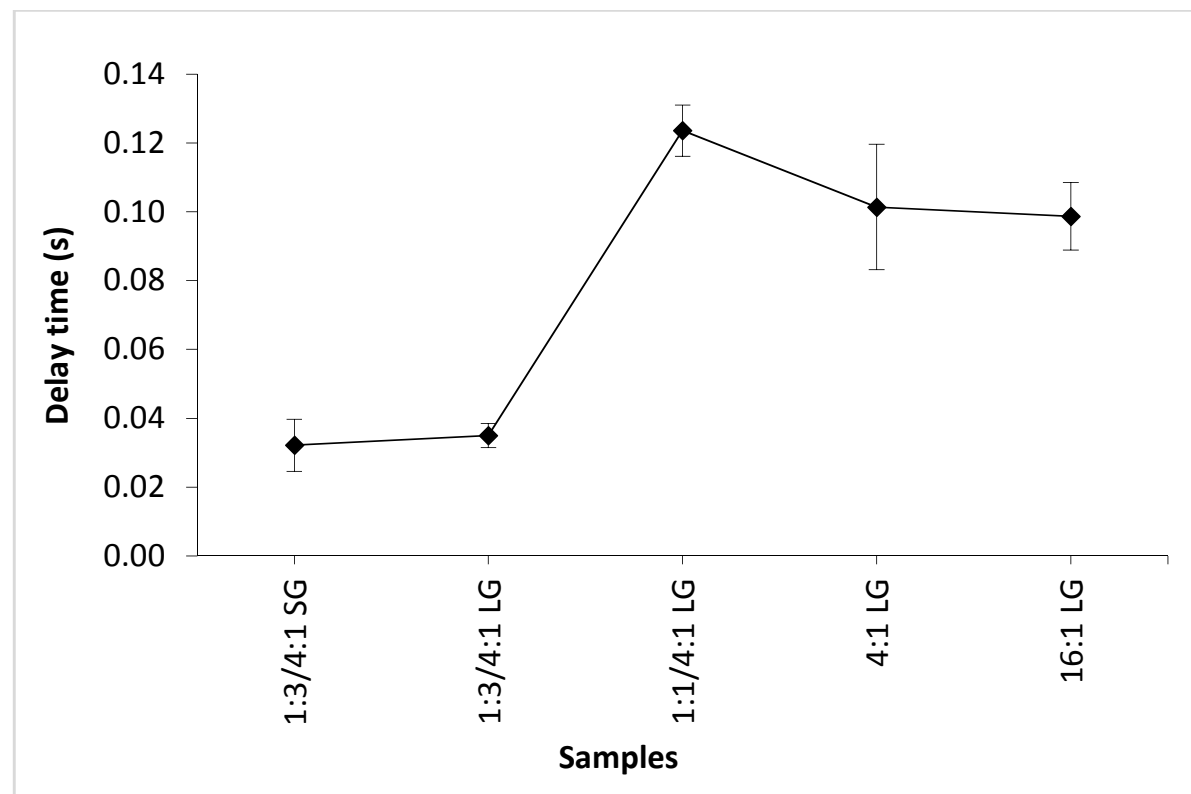

Figure 7. Effect of the fast-crash (S:AS ratio) on ignition delay for GNP-doped RDX (The connecting line is for guidance only).

It is clear that there is not a linear relationship between the S:AS ratio and the delay time for the large GNPs, however it is also clear that the delay time for the fastest initial precipitations (1:3/4:1) is identical whether large or small GNPs were used, indicating that the fastest precipitations reduce ignition delay most effectively. Stronger crashes are known to improve nanoparticle inclusion [20], so the reduced ignition delay is likely to reflect the higher efficiency of the doping procedure, which allows the GNPs to transfer more heat into the RDX rather than the environment and most likely the general smaller sizes of crystal, which will cause differences in the heat exchange distribution within the body of the material. The local temperature within the RDX rises to the required ignition temperature faster in smaller crystals, explaining the reduced ignition delay.

The step trend of the graph indicates a non-linear relationship between the rate of precipitation and ignition delay, the slower precipitations (1:1/4:1, 4:1 and 16:1), all needing greater ignition delay times compared to the $1: 3 / 4: 1$ precipitations, most likely reflecting variations in crystal size among the samples given that slower precipitations tend to produce larger crystals [24]. The fastest precipitation sample using large GNPs exhibited delay times reduced by $65 \%$ compared to the slowest precipitation using the same large GNPs. This indicates that the rate of precipitation is important in understanding the later physical processes, at some point between the 1:1/4:1 and $1: 3 / 4: 1$ precipitation rates, the particle size reduces dramatically - indicating that the precipitation rate and thus crystallite size is more important than GNP inclusion in determining the ignition delay time.

Ignition threshold: Seven samples of the same type were irradiated at each power level, and an ignition rate (percentage) for the sample at the laser power was determined by the number of ignited events $\left(n_{i}\right)$ with a single shot among seven repeated ignition attempts at the same laser power (i.e. $n_{i} / 7 \times 100 \%$ ). Figure 8 shows the rates for each of the samples compared to the laser power. The $70 \%(0.7)$ line is shown for comparison and the ignition thresholds at $\sim 70 \%$ ignition rate are 
summarised in Table 2. Faster initial precipitation (lower S:AS ratios) clearly reduces the power required for ignition, which reflects the better doping efficiency and the resulting greater heat transfer within the RDX.

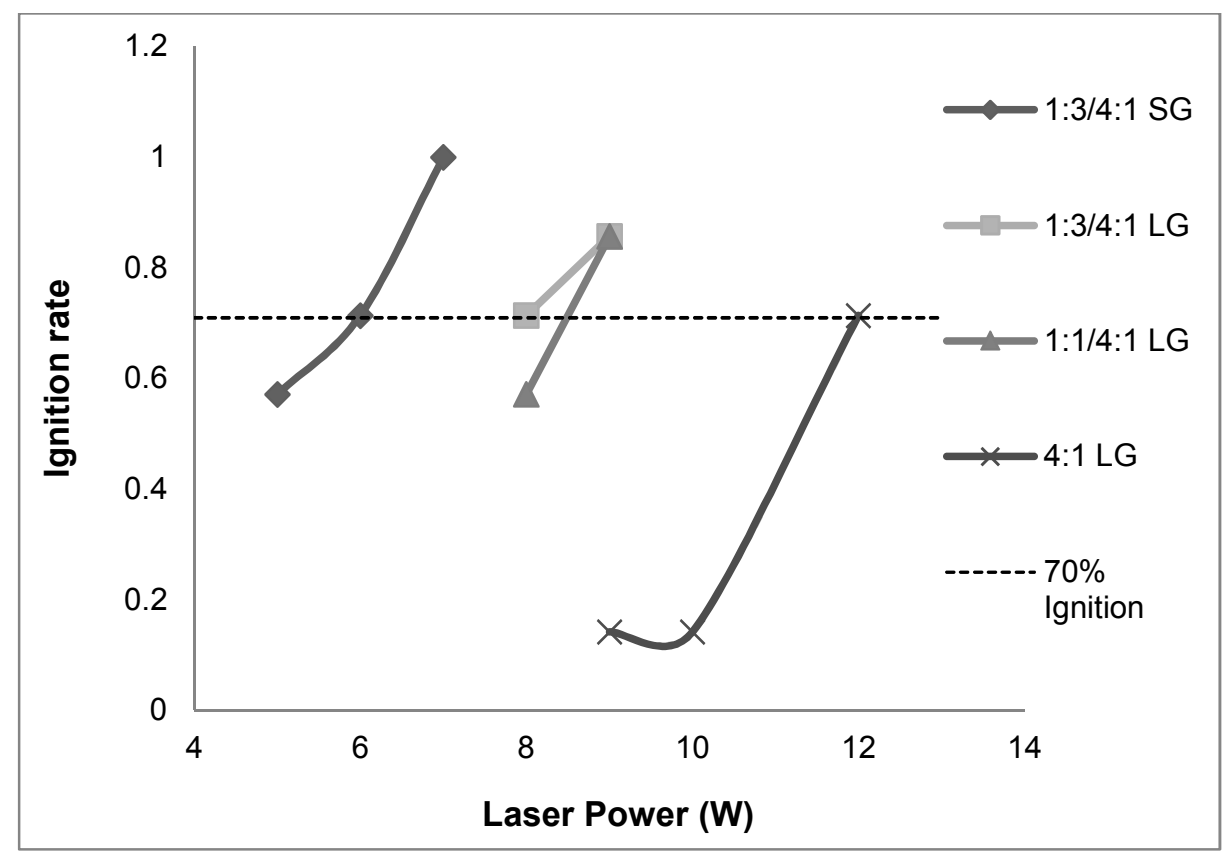

Figure 8. Effect of the fast-crash (S:AS) ratio on ignition threshold for GNP-doped RDX.

Table 2. The laser ignition threshold for $70 \%$ ignition rate.

\begin{tabular}{lcccc}
\hline Samples & $1: 3 / 4: 1$ SG & $1: 3 / 4: 1$ LG & $1: 1 / 4: 1$ LG & $4: 1$ LG \\
\hline Ignition threshold (W) & 6 & 8 & 8.6 & 12 \\
\hline
\end{tabular}

The ignition threshold for crystals doped with small GNPs rather than large GNPs is significantly reduced compared to those containing large GNPs under the same precipitation conditions. This can be ascribed to the relative absorption efficiencies of the different-sized GNPs. Nanoparticles scatter and absorb light, and the ratio of absorption versus scattering depends on size. Smaller nanoparticles absorb more than they scatter and larger nanoparticles show the opposite behaviour [18]. The smaller GNPs thus have a lower ignition threshold because they absorb more laser energy and hence generate more heat. The more efficient absorption by the smaller GNPs is also evident in the sample's darker colour as shown in Figure 4. Both the large and small GNPs were tuned to exhibit peak plasmon resonance at $808 \mathrm{~nm}$ so the lower ignition threshold for the small GNPs is not due to the effect of nanoparticle size on peak resonance. The lower ignition threshold for the smaller GNPs may also in part reflect better doping efficiency due to preferential interactions during the RDX 
crystallisation process. However, this cannot be confirmed without a quantitative method for the analysis of doping, and we conclude that the lower ignition threshold is primarily due to the more efficient absorption of energy by the smaller GNPs. However it is clear that the ignition threshold is much more sensitive to GNP size than it is to the crystallite size.

Large crystal ignition: In examining powder samples for ignition delay and threshold studies, a visual inspection of the burning plate after the experiment clearly indicates the laser initiation, by its leaving of a "bubbled" residue, as shown in Figure 9.

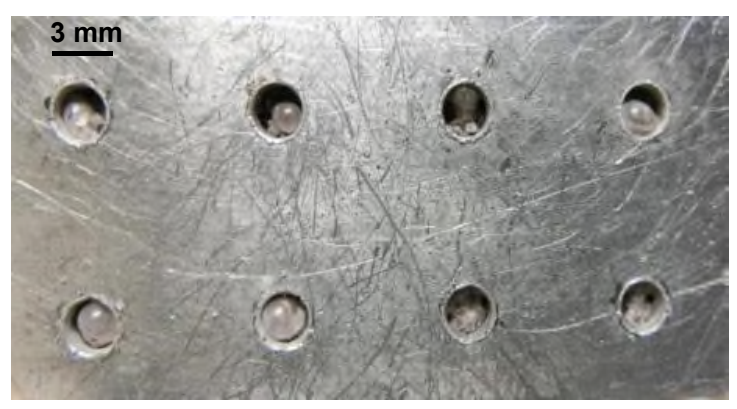

Figure 9. Bubbled RDX residues from ignited 1:1/4:1 large GNP samples. The scale bar represents $3 \mathrm{~mm}$ (hole diameter and depth).

To further study the effect of crystal size, large $(\geq 1 \mathrm{~mm})$ sized single crystals were examined in the same manner. Figures 10 and 11 show representative video stills of the initiation of large crystals grown from S:AS ratios of 16:1 and 1:3/4:1 (least and greatest S:AS ratios). The 16:1 sample in Figure 10 can be seen to ignite from the centre, cracking the crystal in half, implying that the doping process was efficient and that enough GNP was incorporated into the heart of the crystal. In contrast, the 1:3/4:1 sample in Figure 11 ignited much later and ignition was initiated on the surface, leaving the crystal largely intact. This is evidence of poor doping because the nanoparticles appear to be located primarily on the surface of the crystal.
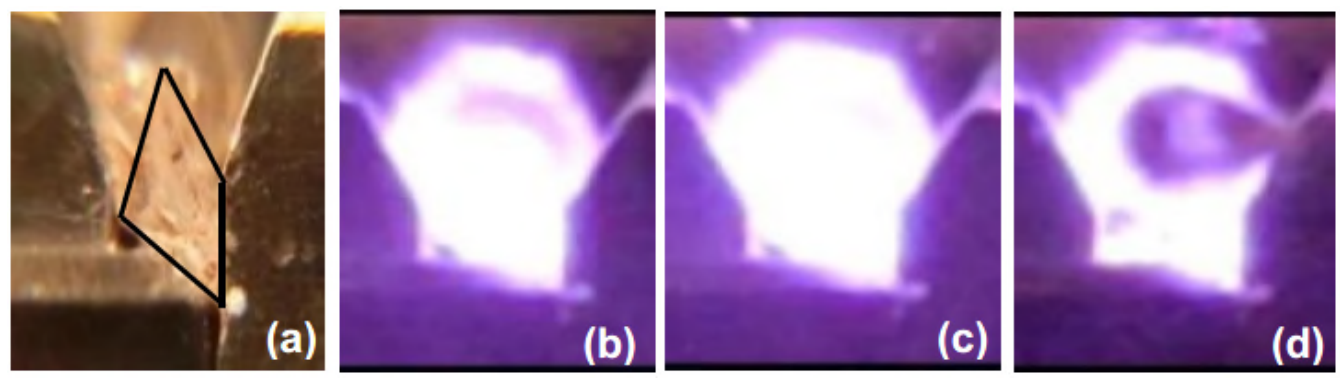

Figure 10. Ignition of a large crystal (outlined) of the 16:1 sample. (a) Prior to ignition; (b) $\mathrm{t}=0 \mathrm{~s}$; (c) $\mathrm{t}=0.010 \mathrm{~s}$ (ignition); (d) $\mathrm{t}=0.037 \mathrm{~s}$. 

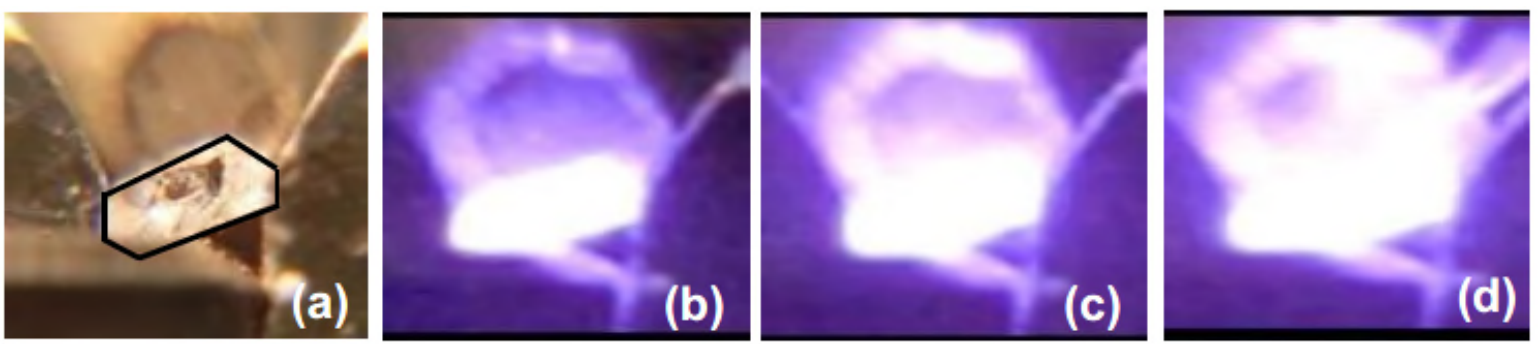

Figure 11. Ignition of a large crystal of the 1:3/4:1 sample. (a) Prior to ignition; (b) $t=$ $0 \mathrm{~s}$; (c) $\mathrm{t}=0.215 \mathrm{~s}$ (ignition). (d) $\mathrm{t}=0.217 \mathrm{~s}$.

In contrast to the powder samples, the large crystals showed no evidence of the RDX bubbling, but either split in two, or fractured where an ignition event occurred. What is also evident is that the relationship between the S:AS ratio and the ignition delay time is the opposite to that observed with the general samples, i.e. for the largest crystals, a slower precipitation led to faster ignition, as measured by high-speed photography and given in Table 3.

Table 3. Comparison of ignition delay times of GNP doped large crystals and powders.

\begin{tabular}{lcc}
\hline Sample & Single crystal mean ignition delay time & $\begin{array}{c}\text { Powder mean ignition delay time } \\
(\mathbf{m s})\end{array}$ \\
\hline $1: 3 / 4: 1$ & 215 & 35 \\
$4: 1$ & 9 & 101 \\
$16: 1$ & 10 & 99 \\
\hline
\end{tabular}

The seemingly contradictory data within Table 3 can be understood in the context of crystal growth and particle size. For single step precipitations, whether small or large, the GNPs are generally dispersed through the crystalline matrix. (see Figures $12 \mathrm{a}$ and $12 \mathrm{~b}$ ). For two-step precipitations, the initial nucleation and growth sites will have a greater ratio of GNPs, than the outer layers, grown after addition of the second RDX/Acetone solution. This will lead to a more uneven distribution of GNPs within the crystal matrices. For small (rapidly precipitated crystals), this does not matter too much, as lots of whole crystals will reside within the laser beam (see Figure 12c) and light scatter/heat loss will be small compared to larger crystals. In the case of larger single crystals of two-step precipitation (Figure 12d), there will be a much larger outer surface covering of crystalline material denuded of GNPs and it may even be possible that the laser light might not impinge upon the GNP rich seed crystal. Hence the delay time increases for these crystals. This would also explain why the largest crystals initiate from the centre of the crystal and the intermediate sized crystals only initiate at the surfaces. 


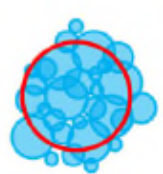

a

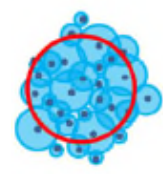

C
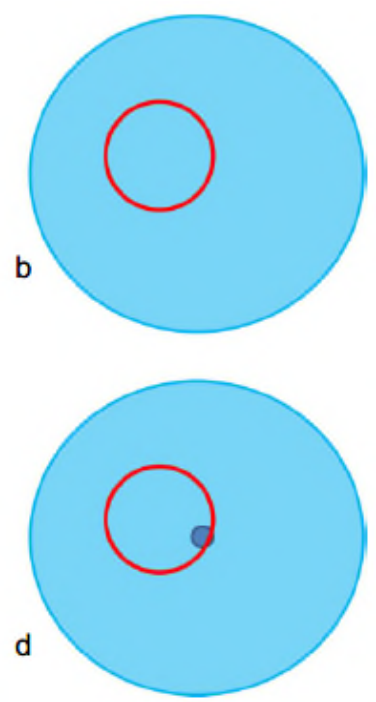

Figure 12. Incident laser beam (red circle) on crystals of various sizes (a) small singlestep precipitated crystals entirely sitting within the beam; (b) large single-step precipitated crystal entirely encompassing the beam; (c) small two-step precipitated crystals entirely sitting within the beam; , dark spots indicating GNP rich seed (d) large two-step precipitated crystal entirely encompassing the beam, dark area indicating the GNP rich seed.

\section{Conclusions}

It can be seen that the inclusion of GNPs allows the laser induced ignition of RDX. The size of GNPs, whether large or small, does not dramatically change the ignition delay time which is more controlled by the crystal size dependent upon the precipitation rate. As a result of the GNPs' effect as nucleating agents, small crystal sizes have good ignition delay times, while for large size crystals, the delay time can increase due to poor doping of GNPs in the two-step growth process. The effect of the different sizes of GNPs are more apparent in the laser power threshold experiments, where the smaller GNP size means lower laser power inputs can be used to effect initiation. Smaller GNPs reduce the power requirements for ignition by $25 \%$ compared to large GNPs. This is because smaller GNPs are better able to absorb the laser energy. The possibility that GNP size alters the plasmon resonance frequency can be discounted because both the large and small GNPs were designed to resonate at 808 $\mathrm{nm}$.

The rate of precipitation is important in generating the appropriate crystal size and it can be seen from the above that this is critical for generating the appropriate delay time. More controlled crystallisation process and downstream processing (e.g. simple sieving) will permit the effect of crystallite size to be accurately determined. Figure 7 shows that there is a step change in delay time with the faster precipitation routes, this leads to the question of whether there is a further step change with even smaller crystal size and could be a route for future study. In particular, given that such energetic materials are often particulates within an amorphous matrix (such as plastic bonded explosives), the ability to ensure an igniting incident beam is vital. 
The GNPs are incorporated within the bulk of the crystals, but the generation of large macrosteps, most likely due to large GNPs acting as pinning sites shows that for preferential ignition properties (short delay time and low power threshold), small GNPs ought to be used. Although the large GNPs do show the shorter delay times, the large macrosteps that occur in this relatively well controlled crystallisation indicates that in less controlled crystallisations (such as those used within industrial manufacture), these will likely engender the formation of large inclusions of mother liquor within the crystal, which then cause a loss of performance. Whether GNPs have any crystallographic relationship with the crystal matrix that they sit in is still an open question and also deserves future study.

The interaction of GNPs and the bulk of a crystal matrix in which it sits, does not lead to straightforward answers. Dependent upon the size, the GNPs can have either no obvious effect upon the materials matrix, or can cause it to be more defective. However, the inclusion of GNPs clearly changes the ignition profile of the energetic material in which it sits. The changes in ignition delay time and power threshold are dependent upon the existence of the GNPs, even if the ignition delay time is also associated with crystal size. It should not be forgotten that the presence of GNPs affect the nucleation and thus growth rate of the crystals.

\section{Acknowledgements}

The authors would like to thank Dr Phil Gill for his valuable advice on sample preparation and Dr Mark Carpenter for his support with optical microscopy. The author (SC) also wishes to thank QinetiQ for sponsorship throughout his MSc course.

\section{References}

[1] Headquarters, US Army Material Command. ACMP 706-179: Engineering Design Handbook Explosive Trains. Alexandria, VA: Headquarters United States Army Materiel Command; 1974.

[2] R. J. Spear, L. D. Redman, J. R. Bentley, MRL-R-881: Sensitization of High Density Silver Azide to Stab Initiation. Melbourne, Victoria: Defence Science and Technology Organisation; 1983.

[3] J. Akhavan. The Chemistry of Explosives. 2nd edn. Royal Society of Chemistry; 2004.

[4] M. G. Wolfson, DSTO-GD-0118: A Guide to Explosives Firing. Melbourne, Victoria: DSTO; 1996.

[5] M. J. Caron, 202-288-2029: Reports of Premature Initiation of Electric Detonators by Radio Frequency Energy. Ontario: Explosives Risk Managers LLC; 2015.

[6] M. H. V. Huynh, M. A. Hiskey, T. J. Meyer, M. Wetzler, Proc. Natl Acad. Sci. U.S.A. 103 (2006) 5409.

[7] P. L. Marinkas, AD-A015 538: Luminescence Properties of RDX and HMX. Dover, NJ: Picatinny Arsenal; 1975. 
[8] A. M. Renlund, P. L. Stanton, W. M. Trott, Laser Initiation of Secondary Explosives. Proceedings of $9^{\text {th }}$ International Symposium on Detonation, 2 (1989), pp. $1118-1127$.

[9] Z. Yan, W. Liu, C. Zhang, X. Wang, J. Li, Z. Yang, X. Xiang, M. Huang, B. Tan, G. Zhou, W. Liao, Z. Li, L. Li, H. Yan, X. Yuan, J. Hazard. Mater. 313 (2016) 103.

[10] Z. Yan, C. Zhang, W. Liu, J. Li, M. Huang, X. Wang, G. Zhou, B. Tan, Z. Yang, Z. Li, L. Li, H. Yan, X. Yuan, X. Zu, Sci. Rep. 6 (2016) 20251.

[11] S. R. Ahmad, M. Cartwright. Laser Ignition of Energetic Materials. 1st ed. John Wiley and Sons, Itd; 2015.

[12] S. D. McGrane, D. S. Moore, Propellants, Explos., Pyrotech. 36 (2011) 327.

[13] X. Fang, W. G. McLuckie, J. Hazard. Mater. 285 (2015) 375.

[14] X. Fang, S. R. Ahmad, Cent. Eur. J. Energ. Mater. 13 (2016) 103.

[15] P. Gillard, L. Courty, S. D. Persis, J.F. Lagrange, C. Boulnois, and I. Gökalp, J. Energ. Mater. 36 (2018) 362

[16] X. Fang, M. Sharma, C. Stennett, P. P. Gill, Combust. Flame. 183 (2017) 15.

[17] X. Huang, M. A. El-Sayed, J. Adv. Res. 1 (2010) 13.

[18] M. Rycenga, C. M. Cobley, J. Zeng, W. Li, C. H. Moran, Q. Zhang, D. Qin, Y. Xia, Chem. Rev. 111 (2011) 3669.

[19] M. W. Knight, N. S. King, L. Liu, H. O. Everitt, P. Nordlander, N. J. Halas. ACS Nano. 8 (2014) 834.

[20] D. A. Reese, S. F. Son, L. J. Groven. Propellants, Explos. Pyrotech. 37 (2012) 635.

[21] J. T. Hagan, M. M. Chaudhuri, J. Mater. Sci. 12 (1977) 1055.

[22] P. J. Halfpenny, K. J. Roberts, J. N. Sherwood, J. Mater. Sci. 19 (1984) 1629.

[23] M. Murugesan, D. Cunningham, J-L. Martinez-Albertos, R. M. Vrcelj, B. D. Moore, Chem. Commun. (2005) 2677.

[24] A. Pant, A. K. Nandi, S. P. Newale, V. P. Gajbhiye, H. Prasanth, R. K. Pandey, Cent. Eur. J. Energ. Mater. 10 (2013) 393. 